\title{
1 A local criterion for ductile fracture under low-triaxiality axisymmetric
}

2 stress states

4 Christopher Smith ${ }^{\mathrm{a}}$, Amit Kanvinde ${ }^{\mathrm{b} *}$, Gregory Deierlein ${ }^{\mathrm{c}}$

${ }^{1}$ Catastrophe Engineering \& Analytics, Berkshire Hathaway Specialty Insurance, Boston, MA, 02110, USA

${ }^{2}$ Department of Civil and Environmental Engineering, University of California at Davis; Davis, CA 95616, 9 USA

$10{ }^{3}$ Department of Civil and Environmental Engineering, Stanford University; Stanford, CA 94305, USA

11 *Email: kanvinde@ucdavis.edu; Tel:530-752-2605

12

13

14 ABSTRACT

16 A local criterion is presented for ductile fracture of metals under a wide range of stress

17 triaxiality. In contrast to previous criteria, whose accuracy is generally limited to the

18 narrower stress ranges over which they were originally validated, the proposed criterion

19 is rigorously supported through (1) hypothesis testing against 352 experiments conducted

20 during previous studies of high- and low-triaxiality fracture, and (2) micromechanical

21 models of the underlying fracture process. The proposed criterion implies a low-

22 triaxiality fracture cutoff at a stress triaxiality of approximately zero, which is supported

23 by experimental test data.

\section{Keywords:}

25 Ductile fracture; Low triaxiality; Steel; Probabilistic fracture mechanics 


\section{1. Introduction}

28 Various models and criteria for predicting ductile fracture initiation are available to

29 evaluate the fracture resistance of metal structures. In contrast to conventional far-field

30 fracture parameters, such as the J-integral or the stress intensity factor $\mathrm{K}$, that are

31 evaluated at some distance away from the crack tip, local models [1] predict fracture in

32 terms of the continuum stress and strain at the point of fracture initiation. Local models

33 are especially useful in situations where large-scale yielding prevents the application of

34 traditional far-field measures, as traditional fracture criteria are limited by assumptions

35 of small scale yielding, monotonic loading, or the presumption of a sharp initial crack

36 [2]. Local fracture criteria are often supported by an understanding of the underlying

37 fracture mechanism and its relationship to the material microstructure. Hence, they are

38 often termed micromechanical models.

39 There is strong evidence that the formation of ductile cracks in polycrystalline 40 metals (e.g., steel) depends strongly on the accumulated plastic deformation, represented

41 by the equivalent plastic strain $\left(\overline{\varepsilon_{P}}\right)$, and on the degree of hydrostatic confinement. The

42 hydrostatic confinement is typically represented by the dimensionless stress triaxiality,

43 defined as the ratio between the mean (hydrostatic) stress and the von Mises (deviatoric,

44 or equivalent) stress $\left(T=\frac{\sigma_{m}}{\sigma_{V M}}\right)$.

45 A significant distinction has been noted [3,4] between the regimes of high and low 46 stress triaxiality, in terms of both the micromechanical fracture mechanism and the

47 empirical measurements of fracture toughness. High values of triaxiality (i.e., $\mathrm{T}>1.5$ )

48 are found in highly constrained regions such as at crack tips, or at the center of necking 49 or notched tension specimens. Low triaxiality conditions are often found at surfaces and

50 protruding corners, where the principal shear stress is high relative to the tensile

51 hydrostatic pressure. Dating back to the 1940's, Bridgman [5] conducted landmark

52 experiments to demonstrate that low triaxiality strongly inhibits fracture initiation.

53 Subsequent studies investigated the effect of triaxiality in more detail, through analytical

54 models of the growth of inter-granular voids [6,7]and tension tests of pre-notched round

55 bars[8,9]. These studies have found strong evidence that under high triaxiality

56 conditions $(\mathrm{T}>1.5)$, the local strain at fracture is related to the triaxiality by a decreasing

57 exponential function (e.g., ${\overline{\varepsilon_{P}}}_{\text {crit }}=\alpha * e^{-B * T}$ ), where the exponent coefficient $\mathrm{B}$ is in 
the range of 1.0 to $2.0[8,10,11]$.

59 More recently, researchers in various engineering disciplines have applied this

60 exponential functional form to conditions outside of those for which it was originally

61 developed. These conditions include cyclic loading typical of earthquakes [2], non-

62 axisymmetric deviatoric stress states [12], and stress states with low $(\mathrm{T}<1.0)$ triaxiality

63 [13]. These efforts have achieved mixed levels of success, especially in the prediction of

64 fracture under low triaxiality conditions.

65 When loaded under high hydrostatic compression, steel samples have been observed 66 to withstand extraordinarily high plastic strains (logarithmic strains over 3.0) without

67 fracturing. This effect was demonstrated by Bridgman [5] in high-pressure test 68 chambers, and Kudo and Aoi [14] in compressive "upsetting" tests (tests of compressive 69 cylinders where fracture occurs after specimen bulging). Building on this evidence, 70 recent research has sought to define a low-triaxiality fracture cutoff below which 71 fracture is very unlikely or practically impossible[4]. Quantifying this type of a cutoff is 72 especially important when low triaxiality stress states are of interest.

73 This paper proposes an improved local criterion for ductile fracture in steel 74 under both high-triaxiality and low-triaxiality conditions. The criterion is supported by 75 objective maximum-likelihood based hypothesis testing and data from 352 experiments 76 reported in two independent previously published studies [5,11]. The next section 77 outlines the sources of data that validate the new criterion, including low-triaxiality 78 experiments [5], high-triaxiality experiments [11], analytical void models [6], and 79 computational void models [15]. Subsequent sections describe a probabilistic 80 framework for the proposed fracture criterion and apply the method of maximum

81 likelihood estimation to compare the proposed and existing models.

\section{2. Current local fracture criteria and their application to low-triaxiality 83 stress states}

84 Examination of dimpled fracture surfaces has indicated that ductile fracture in 85 metals is precipitated by the growth of microvoids (on the order of $10 \mu \mathrm{m}$ ) until the 86 voids coalesce to form a macroscopic crack [16]. Several successful local criteria are 87 based in simulations of the growth of these microvoids. The closely related Stress88 Modified Critical Strain (SMCS) and Void Growth Model (VGM) [17] belong to a 
89 family of "exponential" ductile fracture criteria. The specific form of both criteria are 90 based on analytical derivations by Rice and Tracey [6], who showed that the

91 instantaneous radial growth rate $\left(\frac{d R}{R}\right)$ of a spherical void in an infinite, elastic-plastic

92 continuum is controlled by the stress triaxiality and the rate of applied equivalent plastic

93 strain $\left(\overline{d \varepsilon}_{p}=d \varepsilon_{p}: d \varepsilon_{p}\right)$. For highly triaxial $(\mathrm{T}>1.5)$ and axisymmetric (two identical

94 principal stresses) stress states, Rice and Tracey derived the following expression for the

95 void growth rate (Equation 1). McClintock [7] derived similar results for cylindrical 96 voids.

$$
\frac{d R}{R}=0.283 * e^{1.5 * T} * d \bar{\varepsilon}_{p}
$$

99 If it is assumed that the stress triaxiality in Equation 1 remains relatively constant 100 throughout the growth history of the voids, and that fracture occurs when the voids grow 101 to a critical size, then Equation 1 may be simplified to reveal that that the plastic strain 102 at fracture is inversely related to the void growth rate. Following this, Hancock and 103 Mackenzie [10] proposed the SMCS fracture criterion (Equation 2). The SMCS is 104 referred to as a "critical strain" criterion because fracture is predicted when the strain 105 exceeds a critical value. This type of criterion has been widely used and adapted $[17,18]$ 106 including a more general form by Johnson and Cook [8] that has been adopted in 107 commercial finite element software [19].

$$
\bar{\varepsilon}_{p} \geq \bar{\varepsilon}_{p, \text { crit }}=\alpha * e^{-1.5 * T}
$$

108 Despite the simplicity of the SMCS, large plastic strains are accompanied by 109 evolution of the geometry (e.g., necking) and consequently, the evolution of the local 110 stress state. This invalidates the constant-triaxiality assumption of the SMCS criterion 111 [2], motivating a fracture criterion that considers the entire history of the local 112 parameters. The Void Growth Model (VGM) is designed to account for the loading 113 history by integrating the void growth rate over the local strain history to predict the 114 total void growth. In this model, the void size is assumed to quantify the fracture 115 "damage" (D). The fracture damage calculation for the VGM is shown in Equation 3, 116 and fracture is predicted when the damage exceeds the void-growth capacity, which is 117 considered to be a fundamental material property. 


$$
D=\int_{\bar{\varepsilon}_{p}} e^{1.5 * T} d \bar{\varepsilon}_{p} \geq D_{c r i t}
$$

118 The VGM and SMCS are both based on the same exponential relationship between 119 the void growth rate and plastic strain rate, and were originally developed and applied to 120 predict fracture at high triaxiality (e.g. in the vicinity of cracks and notches). Rice and 121 Tracey [6] briefly extend their derivation of microvoid growth to low triaxiality but 122 focus on a simplified equation valid for $\mathrm{T}>2.0$. Johnson and Cook [8], Rousselier [20], 123 and Panontin [17] all validated an SMCS-type criterion using tests of round 124 circumferentially notched tension specimens, under characteristic triaxiality levels 125 between 1.0 and 2.0. However, the available literature does not support the extrapolation 126 of exponential criteria to low levels of stress triaxiality (i.e., $\mathrm{T}<1$ ).

127 The exponential fracture criteria in Equations 2 and 3 predict rather attainable 128 fracture strains for stress states with low and even negative triaxiality. For example, 129 Equation 3 predicts that under pure compression $(\mathrm{T}=-1 / 3)$ fracture would occur at only 130 four times the plastic strain that would initiate fracture in a standard tension coupon $(\mathrm{T}=$ 131 0.8). However, many experimental and analytical results suggest that ductile fracture 132 becomes increasingly unlikely at these very low triaxiality levels.

133 Bridgman [5], among others [3,21], reports the results of several hundred round 134 tension coupons in a chamber filled with high-pressure hydraulic fluid. While these 135 coupons were pulled in "tension", the externally applied pressure was sufficient to 136 maintain an internal mean stress that was compressive, and thus a negative stress 137 triaxiality. In many of these tests of conventional mild steels, the specimens with 138 negative triaxialities withstood local logarithmic strains in excess of 3.0 (engineering 139 strains over 2000\%) without fracturing. A second experimental example is that of 140 compressive cylinder tests, known as "upset" tests, commonly used for characterizing 141 material properties for metal forming [22]. Upset specimens can be deformed in 142 compression to significant plastic strain levels $\left(\varepsilon_{P}>3.0\right)$. When fracture does occur in 143 the upset compression tests, it only occurs after the specimens "barrel" and the resulting 144 hoop stress increases the stress triaxiality [22,23]. Further examples of this effect are 145 routinely noted in metal-working processes such as wire drawing, where the material 146 can undergo very large plastic strains without fracture, since the metal-working 
147 equipment is designed such that the stress triaxiality remains negative [24].

148 The inhibition of fracture at very low triaxiality has also been suggested by 149 analytical and computational models of microvoid growth. For example, the derivations 150 by Rice and Tracey [6] found the void growth rate to be antisymmetric about the zero 151 triaxiality axis, i.e. plastic strain under a negative triaxiality level will cause voids to 152 shrink at the same rate as the voids would grow if the sign of the triaxiality was inverted

$153\left(\frac{d R}{R * d \varepsilon_{P}}(T)=-\frac{d R}{R * d \varepsilon_{P}}(-T)\right)$. This result suggests that for negative triaxiality levels the

154 void growth and coalescence fracture mechanism is not active. Similarly, computational

155 void cell modeling by Cooke [15] found that groups of neighboring voids, when

156 deformed under negative triaxiality, do not grow or coalesce as observed under positive 157 triaxiality.

158 Thus, an objective of this paper is to provide a fracture criterion that captures the 159 observed ductile fracture behavior at low-triaxiality more accurately than the existing 160 models, while maintaining the accurate fracture predictions provided by the 161 aforementioned exponential models at high positive triaxiality. Other alternatives to the 162 exponential models have been proposed, either based on an adaptation of the 163 exponential equation or an entirely different functional form. For example, efforts to 164 extend the SMCS model to much lower triaxiality levels $(\mathrm{T}<0.5)$ have involved 165 significant modifications. Johnson and Cook [8] found that fitting the exponential 166 function to fracture observations in straight (un-notched) tension coupons, which 167 fracture at moderate triaxiality levels $(\mathrm{T}=0.8)$, requires significant changes to the 1.5 168 value of the exponent coefficient. Ohata and Toyoda [13] observed fracture at the free 169 surface of sharply notched tension specimens, where the fracture strains were much 170 lower than predicted with the SMCS model. Based on their observations, Ohata and 171 Toyoda proposed adding a maximum "capping" strain to the model.

172 In addition to these adaptations of the SMCS model, several researchers have 173 proposed fracture models based on alternative functional forms. As per the original 174 derivations of McClintock [7] and Rice and Tracey [6], the void growth rate was shown 175 to be proportional to a hyperbolic sine function of the stress triaxiality for positive and 176 negative triaxiality levels. This full (hyperbolic sine) form of the equation is written in 177 exponential form in Equation 4. The more commonly used interpretation of this 
178 equation, drops the term with the negative exponent (see Equations 2, 3), which is a 179 reasonable approximation for high triaxiality, but loses the triaxiality range versatility of 180 the original hyperbolic sine functional form.

$$
\frac{d R}{R}=0.283 * d \bar{\varepsilon}_{p} * \sinh (1.5 * T)=0.283 * d \bar{\varepsilon}_{p} *\left(e^{1.5 * T}-e^{-1.5 * T}\right)
$$

181 The popular Gurson model [25] also relies on the hyperbolic sine to express the relationship between void growth (i.e., porosity) and stress triaxiality. In the Gurson 183 model, porosity acts as a surrogate for fracture damage in addition to influencing the 184 constitutive response. In an effort to better model stress regimes with lower triaxiality, 185 Huang [26] reevaluated the analytical solutions of Rice and Tracey for the void growth 186 by accounting for more complex displacement fields (expanding the approximation 187 using more terms) and proposed replacing the void growth rate of Equation 1 with the 188 piecewise expression shown in Equation 5.

$$
\frac{d R}{R}=\left\{\begin{array}{ccc}
0.427 * d \varepsilon_{P} * e^{1.5 * T} & \text { for } & T \geq 1 \\
0.427 * T^{0.25} d \varepsilon_{P} * e^{1.5 * T} & \text { for } & \frac{1}{3}<T<1
\end{array}\right.
$$

189 Kuwamura and Yamamoto [27] studied ductile fracture of round notched bars and 190 tension coupons from four structural steels, and provide an alternative quadratic form, 191 shown by Equation 6. This criterion was based partly on prismatic tension coupons, 192 which fracture at relatively low levels of triaxiality, in contrast with the SMCS criterion, 193 which has typically been calibrated using only notched specimens.

$$
\bar{\varepsilon}_{p, \text { frac }}=C * \frac{1}{T^{2}}
$$

194 Recently, Bao and Wierzbicki [4] also observed the disagreement between the 195 current high-triaxiality models and test data from high pressure and upset tests, 196 proposing another piecewise function for the fracture strain, given by Equation 7. Note 197 that this criterion incorporates a self-described fracture "cutoff" at $\mathrm{T}=-1 / 3$, below which 198 the critical strain is assumed infinite, where fracture is not possible.

$$
\bar{\varepsilon}_{P, \text { crit }}=C *\left\{\begin{array}{ccc}
\frac{1}{T} & \text { for } & T>\frac{1}{3} \\
\frac{1}{T+\frac{1}{3}} & \text { for } & -\frac{1}{3}<T<0
\end{array}\right.
$$

199 The models for ductile fracture initiation described above can be separated into three 200 groups, (1) the SMCS and VGM, which are based on a common underlying 
201 (exponential) functional form and have found wide use in many fields, (2) models that

202 have been adapted from the SMCS or VGM for low triaxiality, and (3) independent

203 model forms. Each of these models were developed from a specific set of experiments

204 or analyses, and has been validated within that narrow context. However, a unified

205 criterion tested against these disparate data sets is not available in literature.

206 Overcoming this limitation is the primary motivation for the proposed criterion

207 described in the next section.

208 As described in the next section, the proposed model is restricted to fracture under 209 axisymmetric tension stress states, which specifically describes the condition where two

210 local principal stresses are identical and are less than the third principal stress. This is a

211 special case of the three-dimensional stress state that is found (among other places) at

212 the center of round and square tension specimens, and so has received a disproportionate

213 level of study. Recent studies have found that the degree of axisymmetry in the local

214 stress state, as represented by the Lode angle, has an influence on the fracture process,

215 although much less than the influence of the stress triaxiality [28,29]. This influence of

216 varying axisymmetry is not mentioned in the development of several of the models

217 presented above $[4,13]$, even though these models are based on experimental data sets

218 which mix axisymmetric and non-axisymmetric test data. For the remainder of this

219 paper, the proposed fracture criteria and supporting data represent fracture under 220 exclusively axisymmetric tension conditions. A more general fracture model is reported

221 by the authors in Smith et al. [29].

\section{Proposed criterion for ductile fracture under axisymmetric stress states}

223 Motivated by the need for a unified fracture criterion for a large range of stress

224 triaxiality, Equation 8 proposes the following local fracture criterion:

$$
D_{\text {crit }} \leq D=\int_{0}^{\bar{\varepsilon}_{P, \text { crit }}}\left(B^{+} * e^{\left(A^{+}\right) * T}-B^{-} * e^{-\left(A^{-}\right) * T}\right) d \bar{\varepsilon}_{P}
$$

225 where, the fracture damage parameter $D$ grows with plastic strain according to a 226 stress-dependent damage rate. Similar to other void growth models, fracture initiation is 227 predicted when the accumulated damage exceeds some critical value, denoted as the 228 fracture damage capacity $D_{\text {crit }}$. The terms inside the integral are the rate form of the 229 fracture criterion, expressed separately in Equation 9: 


$$
\frac{d D}{d \bar{\varepsilon}_{P}}=\left(B^{+} * e^{\left(A^{+}\right) * T}-B^{-} * e^{-\left(A^{-}\right) * T}\right)
$$

230 This criterion differs from the previous VGM by introducing a second exponential 231 term that reduces the damage rate as the stress triaxiality decreases. As shown in Figure 232 1, the implied damage rate decreases to zero as triaxiality tends to zero and becomes 233 negative for triaxiality less than zero. Considering that fracture is predicted by the 234 criterion when the damage exceeds some positive value, a fracture cutoff is implicit at 235 the point where the damage rate (Equation 9) becomes negative. The constants in the 236 equations are $B$, representing the magnitude of the damage rate and $A$, representing the 237 influence of the stress triaxiality on the damage rate. Each set of constants is separated 238 into a positive and negative instance (e.g. $B^{+}$and $B^{-}$) to allow for differences in the 239 damage process under tension and compression. Other researchers have investigated this 240 issue of tension and compression damage to find slight differences in the appropriate 241 constants $[15,30,31]$, but these variations appear to be small relative to the uncertainty 242 involved in their assessment. The separation of these constants does allows the presence

243 and location of the fracture cutoff to be defined parametrically by modifying the ratio $244 B^{+} / B^{-}$and observing the point where the damage rate is zero. As $B^{+} / B^{-}$approaches 245 infinity, the form of the fracture criterion reduces to the VGM.

246 The origins of the proposed functional form lie in the hyperbolic sine models 247 previously discussed by Rice, Tracy, McClintock and Gurson [6,7,25]. The additional 248 treatment of the formulation shown in Equations 8 and 9 serves to bring these models in

249 line with the current understanding of the fracture process. Specifically, flexibility is 250 provided through the constants in the criterion because (1) the theoretical growth 251 predictions are for a single ideal spherical void, whereas actual void growth involves 252 multiple voids with irregular shapes, and (2) flexible constants allow the criterion to 253 account for events in the fracture process (e.g. void nucleation [32] and coalescence 254 [15]) that are somewhat independent of void growth.

\section{4. Evidence to support new local fracture criterion}

256 Sources of experimental data from two different specimen types support the 257 proposed fracture criterion. The first type of specimen is a standard round tension 258 coupon tested in a high-pressure chamber, which is ideal for inducing fracture under 
259 low-triaxiality $(\mathrm{T}<1 / 3)$ conditions. The second type of specimen is a round bar with a 260 circumferential groove cut into it, termed the Cylindrical Notched Tension (CNT) 261 specimen. CNT specimens are commonly used to study fracture under higher triaxiality 262 levels $(1<\mathrm{T}<2)$.

263 Local fracture criteria are further supported through their relation to the micro-scale 264 fracture process. The controlling fracture mechanism in ductile steels is that of void 265 growth and coalescence, which may be studied through analytical and computational 266 models. The proposed fracture criteria will be compared to the most detailed results 267 from each of these analysis methods.

\section{$268 \quad 4.1$ Bridgman high-pressure tests}

269 The most detailed source of experimental data for fracture at very low triaxiality ( $\mathrm{T}$ $270<1 / 3)$ is in the hydrostatically pressurized tension specimens tested by Bridgman [5], 271 who developed an apparatus for conducting round tension tests in a chamber filled with 272 pressurized hydraulic fluid. The applied hydraulic pressure could be maintained at 273 several times the yield stress of the steel, such that when the coupons yielded in tension, 274 the stress triaxiality at their center was negative. Other researchers have conducted 275 similar tension tests with externally applied hydrostatic pressure (e.g. [3,33]). Balzer 276 [34] provides a survey of high-pressure test data and methodology.

277 Test data selected from the Bridgman study include 290 round bars tested at 278 hydrostatic compressive pressure levels between atmospheric and $3000 \mathrm{MPa}$. The set 279 includes eight materials that were designated materials 2 through 9 in the Bridgman 280 report. Bridgman considered multiple heat treatments for each material, and the 281 resulting 33 combinations of material and heat treatment are treated as independent 282 materials in this paper.

283 Figure 2 illustrates the extent of the Bridgman specimens by comparing the plastic 284 strain and the stress triaxiality at the end of each test. Both quantities are reported at the 285 assumed point of fracture initiation, the center of the specimen. As shown in the figure, 286 about half of the specimens did not fracture despite reaching large plastic strain levels. 287 Several qualitative conclusions can be made from this data before further examination.

288 First, there is a significant decrease in the likelihood of observing fracture for specimens 
289 in which the stress triaxiality remains low, an observation which supports the existence

290 of a ductile fracture cutoff. Second, even below the supposed cutoff triaxiality, fracture

291 observations are made in cases where the plastic strain is extremely high. These results

292 can be explained by considering that true strain levels greater than $3.5(350 \%)$ are

293 associated with a 97\% reduction in the cross sectional area of a tension coupon. Such

294 highly deformed specimens are very sensitive to perturbations or eccentricity in the

295 applied loading that might cause inaccurate fracture observations. As such, Bridgman's

296 designation of fracture at these points does not necessarily contradict the fracture cutoff,

297 around $\mathrm{T}=0$, observed in more moderately deformed specimens.

299 4.2 Detailed analysis of the Bridgman data set

300 The fracture criteria described in Equation 8 is based on an integration of the 301 damage rate at the location where a ductile crack may initiate. Evaluating this integral 302 requires the history of the stress triaxiality and plastic strain at that location. In modern 303 tests, the stress-strain history is determined through complementary finite element 304 simulations, but this level of detail is not available for historical test data, where 305 complete load-deformation plots and material parameters are not reported. To consider 306 the effects of the continuum parameter history without the use of finite element analysis, 307 an approximate method is applied, based on derivations by Bridgman. The reported data 308 from the Bridgman tests are limited to the following: the axial stress at initial yielding $309\left(\sigma_{Y}\right)$, necking $\left(\sigma_{\max }\right)$, and fracture $\left(\sigma_{f}\right)$, the test chamber pressure $p$, the reduction in 310 specimen area $\left(A_{i} / A_{f}\right)$, and the ratio between the neck longitudinal radius and tangential 311 diameter $\left(D_{N R} / R_{N}\right)$ measured after the specimen fractured.

312 From this information, three points in the specimen history can be calculated, as

313 illustrated in Figure 3. These points are (A) the initial undeformed condition, (B) the 314 initiation of necking, and (C) the failure of the specimen through fracture or area 315 reduction. The locations of these three points are calculated according to Equations 1031615.

317 Point A: 


$$
\begin{gathered}
T=\frac{1}{3}+\frac{p}{\sigma_{y}} \\
\bar{\varepsilon}_{p}=0
\end{gathered}
$$

318 Point B:

$$
\begin{gathered}
T=\frac{1}{3}+\frac{p}{\sigma_{\max }} \\
\bar{\varepsilon}_{p}=0.2
\end{gathered}
$$

319 Point C:

$$
\begin{gathered}
T=\frac{1}{3}+\frac{p}{\sigma_{f}}+1.4 \ln \left(1+\frac{D_{N R}}{4 R_{N}}\right) \\
\bar{\varepsilon}_{p}=\ln \frac{A_{i}}{A_{f}}
\end{gathered}
$$

320 Equations 10 and 11 calculate the point of initial yielding in the coupon. For tests 321 without a reported initial yield stress, the stress at the point of maximum load $\left(\sigma_{\max }\right)$ 322 was substituted for the yield stress. The externally applied pressure is negative 323 (compressive) and remained constant throughout each test.

324 The initiation of necking in the tension specimens generally coincides with the point 325 of maximum load, and represents an important change in the deformation mechanism 326 (from uniaxial tension and distributed yielding to a localized and sharpening notch). The 327 conditions at the initiation of necking are determined from the point of maximum load 328 by Equations 12 and 13, where Bridgman [5] found the plastic strain to be consistently 329 about 0.2 at the initiation of necking.

330 Bridgman developed an analytical relationship between the geometry of the necked 331 section (i.e. notch radius and diameter) and the internal stress state. Using modern 332 finite element tools, Bao and Wierzbicki [4] proposed a refined version (Equation 14) 333 of the Bridgman equation, accounting for the effects of strain hardening and non334 uniform stress fields across the neck. Assuming a relatively smooth strain distribution 335 across the notch root (validated experimentally by Bridgman), the plastic strain at the 336 center of the specimen is related to the reduction in cross-sectional area by Equation 33715.

338 By interpolating between the above points, the approximate evolution of the 339 triaxiality-strain relationship, shown in Figure 3, was constructed for each of the 
340 Bridgman specimens. In the subsequent sections, this history will be used to evaluate the 341 proposed damage-based fracture criteria (e.g. Equation 8).

\section{$342 \quad 4.3$ Cylindrically Notched Tension data set}

A database of Cylindrically Notched Tension (CNT) tests and standard round

344 tension bar tests, conducted by Kanvinde and Deierlein [30] and Myers et al. [11], and 345 compiled in the report by Myers et al. [11], is used to evaluate the fracture models at 346 high stress triaxiality. A total of 62 CNT specimens and 5 standard round tension 347 coupons are used for this analysis.

348 The stress triaxiality and plastic strain in the smooth tension specimens were 349 calculated using Equations 10 through 15. Myers calculated the corresponding values

350 for the CNT specimens using finite element simulations. In contrast to the smooth 351 tension coupons, where the stress state changes due to the evolution of the neck 352 geometry, the stress triaxiality in CNT specimens is driven by the preexisting notch and 353 remains relatively constant throughout each test. The triaxiality and strain conditions at 354 the end of each test are shown in Figure 4.

\section{4.4 Supporting micromechanical evidence for fracture criteria}

356 4.4.1 Analytical void mechanics

357 Micro-scale models of the ductile fracture process (e.g. void growth and 358 coalescence) provide strong evidence to support ductile fracture criteria. Following the 359 original work of Rice and Tracey [6] discussed in Section 2, void growth models have 360 been extended to more complex materials and stress states by Huang [26] and others. 361 Specifically, Huang reevaluated the solutions for the void growth rate by expanding the 362 Rice-Tracey approximation using more terms to account for more complex displacement

363 fields around the voids. Huang proposed replacing the Rice and Tracey void growth rate 364 (Equation 1) with the piecewise equation shown in Equation 16.

$$
\frac{d R_{v}}{R_{v}}=\left\{\begin{array}{ccc}
0.427 d \varepsilon_{P} e^{1.5 T} & \text { for } & T \geq 1 \\
0.427 T^{0.25} d \varepsilon_{P} e^{1.5 T} & \text { for } & \frac{1}{3}<T<1
\end{array}\right.
$$

365 Compared to the findings of Rice and Tracey, the Huang solution finds a general 366 increase in the void growth rate, along with a more complex solution for triaxiality 
367 levels as low as one-third (whereas Rice and Tracey limited their solution to triaxiality 368 levels above 2.0). It should be noted that Equation 16 is a prediction of the average 369 (volumetric) void growth rate. As the lower hydrostatic tension provided by lower stress

370 triaxiality levels causes prolate void growth, the volumetric void growth may become

371 less correlated with fracture initiation at lower triaxiality.

$372 \quad$ Figure 5 illustrates the agreement between the proposed damage growth rate in

373 Equation 9 and the void growth rates derived by Rice and Tracey [6] and Huang [26].

374 Both of these results for the void growth rate agree well with the proposed damage 375 model.

\section{4.4.2 Computational void models}

377 There are several assumptions inherent in the analytical models for void growth 378 proposed by Rice and Tracey [6] and Huang [26] that inhibit their application at low 379 stress triaxiality levels. First, these models only incorporate the void growth stage of the 380 fracture process. Other studies have shown (1) that a relatively small amount of void 381 growth is required to cause strain localization and void coalescence [29,35,36], and (2) 382 that the critical void growth level is not necessarily independent of the stress triaxiality 383 [15]. As a result, models based purely on void growth predictions may fail to capture 384 significant parts of the fracture process. Second, the assumption of spherical void 385 growth is violated at low triaxiality levels, as voids are observed to grow into long 386 prolate shapes. This aspherical effect may decrease the ability of the average void radius 387 (predicted by the Rice-Tracey equation) to predict fracture.

388 Finite element simulations of void growth and coalescence seek to avoid these issues 389 by directly modeling the micro-scale deformations of the void and surrounding matrix 390 [37-39]. Cooke [15] conducted two such analyses with an emphasis on the low391 triaxiality fracture process which are useful in this application. The first set of 392 simulations measured the volumetric growth rate of a single void that was deformed 393 under various levels of stress triaxiality. As indicated in Figure 6, the void growth rates

394 are closely represented by a model following the form of Equation 9. The simulated 395 void growth rate decreases as the stress triaxiality decreases, becoming negative for 396 values of the stress triaxiality which are below zero. These simulations validate the void 397 growth predictions of Rice and Tracey for low-triaxiality conditions, but they do not 
consider the effects of void interaction on the coalescence and fracture process, an effect that may be more significant at low triaxiality levels.

400 In a second set of simulations, Cooke modeled a multi-void matrix that was loaded

401 until the point of void coalescence. The results from these simulations are shown in

402 Figure 7. The strain required to cause the voids to coalesce increases as the applied 403 stress triaxiality decreases (Figure 7a). Void coalescence is indicated at the micro-scale 404 by the onset of unstable void growth and material softening (Figure 7b). For triaxiality 405 levels below 0.3 , no void coalescence was observed before numerical nonconvergence 406 forced the termination of the analysis.

\section{4.5 Probabilistic evaluation of the new fracture criteria}

408 Ductile fracture in metals is a random process, owing to uncertainty in the local 409 stress and strain fields, material properties, and the fracture process itself. Typical 410 fracture models provide only a deterministic prediction of fracture, where the inherent 411 randomness is considered implicitly using regression to minimize the error between 412 model predictions and observed data. These deterministic models provide good 413 predictions for a single uniform material with high quality test data under monotonic 414 loading [40,41]. However, the challenges in fitting a deterministic fracture model to 415 complex cyclic test data have recently been demonstrated by (1) Myers et al. [42] who 416 applied fracture models to specimens with inhomogeneous material properties around 417 welded connections and (2) Smith et al. [29] in specimens with sharp gradients of local 418 parameters caused by low triaxiality and complex specimen geometry.

419 To account explicitly for the uncertainty in fracture predictions, and to facilitate 420 more robust calibration, it is necessary to formulate and calibrate the fracture criterion in 421 a probabilistic sense [42]. A probabilistic formulation, first proposed by Myers et al. 422 [42], is based on the assumption that the combined uncertainty can be treated as a 423 random distribution of the fracture capacity $\left(D_{\text {crit }}\right)$. While the true uncertainty in 424 fracture predictions can be attributed to several sources (e.g., experimental error, 425 material properties, or the fracture process itself), treating these sources separately 426 would require an unrealistic amount of test data and analyses. In the "lumped 427 uncertainty" model, validated by Myers et al. [42], the evolution of the fracture damage 
428 (D) can still be calculated deterministically (i.e. Equation 8), producing a single 429 deterministic value to be compared with the stochastic damage capacity.

430 Recall that in the deterministic formulation of the fracture criteria (Equation 8), 431 fracture initiation is predicted at the instant that the fracture damage $(D)$ exceeds the 432 fracture capacity $\left(D_{\text {crit }}\right)$. Alternatively, in the probabilistic formulation of the criteria, 433 there is a non-zero probability of fracture initiation at all levels of the fracture damage $434(D)$. The associated probability distribution of the fracture capacity is a random variable 435 defined as $\widetilde{D_{\text {crit }}}$, and is assumed to be normally distributed [42], with mean $\mu_{D}$, standard 436 deviation $\sigma_{D}$, and coefficient of variation $\operatorname{COV}_{D}=\frac{\mu_{D}}{\sigma_{D}}$.

437 Maximum likelihood estimation (MLE) is a commonly used tool for estimating the 438 parameters of a probabilistic model[43]. MLE is a method of parameter estimation, and 439 operates by searching for the model parameters that are most likely given a set of 440 observations. In the context of this study, the observations may include any physical or 441 computational test whose result (e.g., a measured or simulated fracture event) can be 442 predicted by the fracture model. The most likely parameter set is that which maximizes 443 the probability of the observed results. For statistically independent tests, the likelihood 444 function is written as:

$$
L(\text { parameter set |observation set })=\prod P\left(\text { obs }_{n} \mid \text { parameter set }\right)
$$

445 The equation determines the likelihood that a candidate parameter set is accurate 446 given a set of observed data (which may be the results of physical or computational 447 experiments). This likelihood is equal to the product of the probability of making each 448 observation, were the candidate parameter set true. Per the above description of the 449 probabilistic fracture criteria, the probability of making such an observation is equal to 450 the value of the probability density function $\left(\widetilde{D_{c r i t}}\right)$ at the value of $D$ where fracture was 451 observed. Note that $D_{\text {crit }}$ is not directly observable, since it is derived from FE 452 simulations. However, for the purposes of maximum likelihood estimation, it may be 453 treated as such since it bears a unique correspondence with the observable quantity (i.e., 454 failure displacement) and is consequently a unique indicator of the failure displacement. 455 For example, consider the results of Bridgman's high pressure tests on one material, 456 reported as steel \#2, heat treatment \#7 by Bridgman [5]. The local history of the stress 
457 triaxiality and plastic strain in two typical specimens are determined by Equations 10458 15, and this history is input into the fracture criteria (Equation 8) to evaluate the fracture 459 damage parameter $(D)$ for each test. Figure 8 illustrates the evaluation of the likelihood 460 function for two tests of the material. The upper half of the figure shows the evolution, 461 in triaxiality-strain space, of each of the tested specimens as determined by Equations

462 10-15. The lower half of the figure shows the same tests in triaxiality-damage space, 463 where the damage is determined according to the proposed fracture criteria.

464 Given an assumed distribution of the material fracture toughness (indicated by the 465 normal probability distributions in the figure), the probability of observing a given test 466 is proportional to the Probability Density Function (PDF) of the fracture toughness 467 distribution evaluated at the end of the test: $P\left(o b s_{n}\right)=f_{\widetilde{D}}(D)$, where $D$ is the 468 accumulated fracture damage calculated at the end of the test.

469 Figure 8 shows one test that fractured and one test that did not fracture, even though 470 the second was pulled to a higher level of strain. Aside from representing actual 471 measured data, this second example is also an important observation that can be tested 472 against the candidate fracture criteria. The probability of not observing fracture by the 473 end of one of these tests is equal to the complement of the CDF at the end of the test, i.e. $474 P\left(o b s_{n}\right)=1-F_{\widetilde{D}}(D)$. Then, according to Equation 17, the likelihood of the assumed 475 parameter set given the two tests shown in the figure is equal to the product of the 476 probability of seeing the observed outcome in each test. As illustrated in this example, 477 inclusion of non-fracture (survival) data through maximum likelihood estimation are 478 important contributors to the outcome - which is why this practice is common in clinical 479 trials [44]. Using the MLE method, the search for the fracture model parameters that 480 maximize this likelihood is typically done using numerical optimization.

481 All of the test observations described in the preceding section were evaluated by a 482 similar procedure. The physical observations from the CNT fracture tests mirror those 483 of the Bridgman tests, where the plastic strain, triaxiality, and resulting damage history 484 are calculated for each specimen.

485 In the computational models of void coalescence, the triaxiality and strain history 486 (and thus the damage history) are imposed by the boundary conditions of the finite 487 element model. The "observation" of fracture initiation is recorded at the point of void 
488 coalescence, as measured by the point that the stiffness of the finite element model 489 becomes negative. The modeling parameters that result in the six different curves in 490 Figure 7a represent six separate materials, and the results are analyzed in the same way 491 as separate materials in the high-pressure or CNT experimental data sets.

492 Fracture "observations" are made from the analytical models of void growth by 493 selecting a critical void size which is assumed to represent fracture initiation. Virtual 494 experiments are conducted to evaluate the critical plastic strain at many values of the 495 stress triaxiality. This critical plastic strain and stress triaxiality are combined to 496 calculate the observed damage index $(D)$ at fracture, per Equation 8.

497 The distribution of the fracture toughness parameter $\left(\widetilde{D_{\text {crit }}}\right)$ is a material property 498 that must be separately calibrated for each material. However, the other parameters in 499 the fracture model (i.e. $A^{+}, A^{-}, B^{+}, B^{-}$) are considered to be fairly consistent for all mild 500 steel materials. Other researchers have similarly found these parameters to be relatively 501 material invariant[17,18]. Therefore, calibrating the individual fracture toughness 502 parameters and the model shape parameters requires a two-step process.

503 For a candidate set of characteristic parameters, (e.g. the familiar VGM: $D=$

$504 \int e^{1.5 T} d \varepsilon_{p}$ ) we calibrate the fracture model to each material using MLE. The likelihood

505 returned from this calibration indicates the likelihood that the candidate set of model 506 generic parameters is accurate for each material. Taking the product of the likelihood 507 value for each material yields the combined likelihood that the candidate model 508 parameters are valid given the results of all of the materials and data sources. A 509 mathematical optimization routine is used to find the model parameters that maximize 510 this combined likelihood.

\section{$511 \quad 4.6$ Calibration and discussion of model form}

512 Within the Maximum Likelihood framework described in the previous section, the 513 likelihood value may be used to both calibrate the internal parameters of a proposed 514 fracture criteria and evaluate the relative accuracy of multiple independent criteria. In 515 this way, a wide variety of fracture criteria can be compared objectively. The following 516 figures and table will illustrate the calibration of the newly proposed fracture criteria in 517 Equation 8, and demonstrate the improvement in fracture prediction relative to the 
518 models available in literature. Following the approach described in the previous section,

519 these comparisons will be made in terms of the likelihood of a given model being 520 correct given the available supporting evidence.

521 It is convenient to discuss the likelihood in terms of its logarithm, as they are both

522 maximized under the same conditions and the so-called log-likelihood produces more 523 computationally tractable values. The log-likelihood can be interpreted by considering

524 that for a given data set, a candidate prediction model that increases the log-likelihood

525 by 1.0 is 10 times more likely than the alternative. However, one is cautioned against

526 placing too much weight in the absolute magnitude of the log-likelihood values, as they

527 are dependent on many modeling assumptions and the diversity of the calibration data

528 set. In developing a robust criterion, a reasonable fit to a large set of empirical fracture

529 observations is more important than a perfect fit to a theoretical model or a limited data 530 set.

\section{4.6.1 Calibration of the proposed fracture criterion}

532 Figures 9 and 10 demonstrate the calibration of the proposed fracture criteria 533 (Equation 8) within the MLE framework. In both figures, the log-likelihood of 534 observing each data source (e.g. high-pressure test data, computational void models) is 535 indicated as a function of a parameter of the fracture criteria. The most likely version of 536 the fracture criteria is that which maximizes the likelihood of making all of these 537 collected observations, judged qualitatively as the likelihoods have slightly different 538 maxima. Figure 9 illustrates the likelihood of observing each data source as a function 539 of the fracture cutoff location (mapped to the ratio $\mathrm{B}+/ \mathrm{B}-$ ), while holding the value $540 A=1.5$, with two implications. First, the most likely value for the fracture cutoff, when 541 separately considering each source of observations, falls in the range $(-0.1<\mathrm{T}<0.15)$. 542 Second, the CNT tests and computational void cell models are relatively tolerant of 543 fracture cutoffs that are highly negative (tending in the extreme to the traditional 544 exponential model). This is the expected result, as the CNT and computational data sets 545 mainly contain data for triaxiality greater than one-half. The choice of fracture cutoff 546 has little influence on the shape of the fracture criteria for these higher triaxiality levels.

547 Figure 10 shows the variation in the log-likelihood for values of the exponential 548 parameter $A$ while holding the value $\left(B^{+} / B^{-}=1.0\right)$. With the exception of the high 
549 pressure tests, the plots suggest that the mostly likely value for the $A$ parameter falls 550 between 1.2 and 1.7, a range consistent with other reported values under different test 551 conditions. The likelihood of observing Bridgman's high-pressure test data, however,

552 was almost unaffected by the choice of the $A$ parameter. This is a consequence of the

553 fact that Bridgman's high-pressure test data contained almost exclusively triaxiality 554 levels below $\mathrm{T}=0.8$, such that the shape of the fracture criteria in this region is driven 555 mostly by the presence and location of the fracture cutoff, rather than the $A$ parameter.

556 A separate parametric study was conducted to explore using separate values for the 557 constants $A^{+}$and $A^{-}$. Decoupling the values had minimal impact on the overall 558 likelihood, owing to the relative lack of ability in this data set to separate the rates of 559 positive and negative damage accumulation. Cyclic test data and simulations would 560 have more statistical power in this regard.

561 Based on the results of the two parametric calibrations described above, and 562 considering the uncertainty and relative value of the various data sources, reasonable 563 values for the model shape parameters were selected $\left(A^{+}=A^{-}=1.3 ; B^{+} / B^{-}=1.0\right)$

$$
D=\int\left(e^{1.3 * T}-e^{-1.3 * T}\right) d \bar{\varepsilon}_{P}
$$

564 as shown in Equation 18.

565 These values were chosen to fall within the range of values which maximized the 566 likelihood of observing each data set, while giving preference to matching the 567 observations from physical experiments rather than theory or simulations. The 568 calibrated value of the $A$ parameters is unsurprising, and fits within the range of 569 historical values for similar parameters. The calibrated value of $B^{+} / B^{-}=1.0$ indicates 570 the existance of a fracture cutoff at a stress triaxiality level of zero. This cutoff value is 571 higher than that proposed by Bao and Wierzbicki [4], owing to the fact that that their 572 study did not consider the history effect on fracture damage, but the cutoff location is 573 identical to the fracture cutoff proposed by Reddy et al. [24] for wire drawing 574 operations.

\section{4.6.2 Comparison of the proposed fracture criterion with alternatives}

576 Table 1 shows a comparison of the proposed fracture criterion with several other 577 models found in the literature. The values reported are the log-likelihood, relative to the 
578 proposed model, of each model for each source of supporting data. Therefore, negative 579 values in the table indicate that the specified model is less likely than the proposed 580 criterion, Equation 18; and positive values indicate that the specified model is more 581 likely than the proposed criterion.

582

583

\begin{tabular}{ll}
\hline$D=\int e^{1.3 T}-e^{-1.3 T} d \varepsilon_{P}$ \\
\hline$D=\int e^{1.5 T} d \varepsilon_{P}$ \\
\hline$\varepsilon_{p, \text { crit }}=\alpha e^{-1.5 T}$ \\
\hline$\varepsilon_{p, \text { crit }}=C T^{-2}$ \\
$\varepsilon_{p, \text { crit }}=$ \\
$\left\{\begin{array}{cc}T<0 & C\left(T+\frac{1}{3}\right)^{-1} \\
0<T<\frac{1}{3} & \ldots \\
T>\frac{1}{3} & C T^{-1}\end{array}\right.$
\end{tabular}

584

Analytical void growth

586 When compared to the analytical void growth relationship by Huang [26], the 587 SMCS and VGM are somewhat more likely than the newly proposed criterion. This is an expected result, as derived void growth relationships form the basis for these models.

589 The proposed criterion is significantly more likely than the Kuwamura and Yamamoto

590 [27] and Bao and Wierzbicki [7] models, as they have an independent functional form 591 from the analytical void growth predictions.

$592 \quad$ FEM void coalescence

593 The available observations of computational void coalescence [15] tend to parallel 594 the analytical void growth predictions, except that they also simulate the micro595 mechanical effects of void shape change and coalescence. As indicated by the negative

596 likelihood data, the proposed model is more closely aligned to the observed FEM void 597 coalescence than any of the other four models.

$598 \quad$ CNT tests

599 Recall that the CNT data set includes both CNT specimens (triaxiality at fracture 
600 between 1.0 and 2.0) and standard tension coupons (triaxiality at fracture between 0.5

601 and 0.8). The VGM and SMCS were developed for triaxiality levels above 1.0, and they

602 are found to be less likely than the proposed model or the other two empirical models.

603 In contrast, Kuwamura and Yamamoto developed their model empirically from test data

604 of tension coupons and CNT specimens, and thus, it is not surprising that their model

605 has slightly better correlation with the proposed model. The proposed model also

606 provides a higher likelihood than the Bao and Wierzbicki model.

$607 \quad$ High-pressure tension tests

608 An important motivation of this paper is to address the conflict between existing 609 fracture criteria and the very low-triaxiality test data published by Bridgman, among

610 others. As evidenced by the likelihood parameters in the last column of Table 1, the

611 proposed fracture criterion is a significant improvement over other models to simulate

612 fracture under low-triaxiality. Compared to the proposed model, the VGM is the second

613 most likely model of those investigated, and is the only other damage-based criteria. As

614 discussed earlier, critical strain based criteria are expected to be less reliable for lower

615 triaxiality levels, due to significant variation in the fracture damage rate which those

616 models cannot capture. Of the remaining three critical strain based criteria, Bao and

617 Wierzbicki [4] as well as Kuwamura and Yamamoto [27] are more likely than the

618 SMCS, which does not incorporate a fracture cutoff.

619 As indicated by the table, the newly proposed criterion is shown to have the highest 620 likelihood in predicting both the experimental test data and the theoretical 621 micromechanical observations.

\section{5. Conclusions}

623 This paper has examined the use of local criteria to predict fracture under 624 axisymmetric conditions, including the consideration of the historical justification for 625 such models and their deficiencies. Models for ductile fracture under axisymmetric 626 stress conditions have traditionally been based on the idea that material damage is 627 proportional to an exponential function of the stress triaxiality. These exponential 628 models are particularly important because in addition to their original use for 629 axisymmetric high-triaxiality conditions, they have been extended to alternative 
630 conditions including cyclic loading [2] and non-axisymmetric stress conditions [18].

631 A significant limitation of exponential fracture models is their failure to capture the 632 low-triaxiality fracture cutoff, a triaxiality level below which fracture cannot occur. 633 Evidence from multiple data sources that have been reviewed in this paper suggests the 634 cutoff to occur at a triaxiality level of zero. This implies that ductile fracture is 635 impossible without the presence of tensile hydrostatic pressure (positive triaxiality).

636 The objectives of this study are to find a fracture damage model that (1) closely 637 approximates the results of established models at high stress triaxiality, (2) captures the 638 effect of the low triaxiality fracture cutoff, 3) provides flexibility to allow accurate fits 639 to higher quality data sets, and 4) requires relatively few calibration parameters. These 640 objectives are achieved through the adaptation of the void growth model originally 641 proposed by Rice and Tracey based on the hyperbolic sine of the stress triaxiality. The 642 model was further adapted to accommodate features such as dissimilar void growth and 643 shrinkage rates. The proposed model (Equation 8 and 9) contains four parameters, three 644 of which are shown to be fairly constant across various steel material tests. 645 Recommended values of these parameters are determined based on a combined analysis 646 of the available data. This results in a criterion (Equation 18) with only one parameter, $647 D_{\text {crit }}$, that needs to be calibrated for specific materials.

648 The rediscovery of the hyperbolic sine based fracture prediction model that was 649 proposed by Rice and Tracey in 1969 is an interesting development. The Rice-Tracey 650 exponential model has previously been adapted to simulate fracture under cyclic loading 651 [2] and low-triaxiality stress states [28], including the effect of the triaxiality fracture 652 cutoff [4]. The hyperbolic sine formulation by Rice and Tracey encompasses these 653 objectives in a simple and theoretically justified form. Only minor modification of their 654 model is necessary to fit it accurately to a diverse set of experimental data.

\section{Acknowledgments}

656 This research was supported by the National Science Foundation under Grant 657 Number CMMI-0825339 (NSF Project \#0825339). Any opinions, findings, and 658 conclusions or recommendations expressed in this paper are those of the authors and do 659 not necessarily reflect the views or policies of the National Science Foundation. 


\section{References}

661 [1] C. Berdin, J. Besson, S. Bugat, R. Desmorat, F. Feyel, S. Forest, et al., Local

662 Approach to Fracture, Les Presses de l'École des Mines, 2004.

663 [2] A.M. Kanvinde, G.G. Deierlein, Cyclic Void Growth Model to Assess Ductile

664

665

666

667

668

669

670

671

672

673

674

675

676

677

678

679

680

681

682

683

684

685

686

687

688

689

690

691

692

693

694

695

696

697

698

699 Fracture Initiation in Structural Steels due to Ultra Low Cycle Fatigue, J. Eng. Mech. 133 (2007) 701.

[3] A.S. Kao, H.A. Kuhn, O. Richmond, W.A. Spitzig, Tensile fracture and fractographic analysis of 1045 spheroidized steel under hydrostatic pressure, J. Mater. Res. 5 (1990) 83-91.

[4] Y. Bao, T. Wierzbicki, On the cut-off value of negative triaxiality for fracture, Eng. Fract. Mech. 72 (2005) 1049-1069.

[5] P.W. Bridgman, Studies in large plastic flow and fracture, Harvard University Press, 1964.

[6] J.R. Rice, D. Tracey, On the Ductile Enlargement of Voids in Triaxial Stress Fields, J. Mech. Phys. Solids. (1969).

[7] F.A. McClintock, A criterion for ductile fracture by the growth of holes, J. Appl. Mech. 35 (1968) 363.

[8] G.R. Johnson, W.H. Cook, Fracture characteristics of three metals subjected to various strains, strain rates, temperatures and pressures, Eng. Fract. Mech. 21 (1985) 31-48.

[9] B. Marino, F. Mudry, a. Pineau, Experimental study of cavity growth in ductile rupture, Eng. Fract. Mech. 22 (1985) 989-996.

[10] J. Hancock, A. Mackenzie, On the mechanisms of ductile failure in high-strength steels subjected to multi-axial stress-states, J. Mech. Phys. Solids. 24 (1976) 147160.

[11] A.T. Myers, G.G. Deierlein, A.M. Kanvinde, Testing and Probabilistic Simulation of Ductile Fracture Initiaion in Structural Steel, Blume Cent. Rep. (2009).

[12] Y. Bao, T. Wierzbicki, On fracture locus in the equivalent strain and stress triaxiality space, Int. J. Mech. Sci. 46 (2004) 81-98.

[13] M. Ohata, M. Toyoda, Damage concept for evaluating ductile cracking of steel structure subjected to large-scale cyclic straining, Sci. Technol. Adv. Mater. 5 (2004) 241-249.

[14] H. Kudo, K. Aoi, Effect of compression test condition upon fracturing of a medium carbon steel, J. Japanese Soc. Technol. Plast. (1967).

[15] R.J. Cooke, Micro-Mechanical Simulation of Ductile Fracture Processes in Structural Steel, University of California at Davis, 2015.

[16] T.L. Anderson, Fracture mechanics: fundamentals and applications, Third Edit, CRC Press, Boca Raton, FL, 2005.

[17] T.L. Panontin, The relationship between constraint and ductile fracture initiation as defined by micromechanical analyses, ASTM, 1994. 
700

701

702

703

704

705

706

707

708

709

710

711

712

713

714

715

716

717

718

719

720

721

722

723

724

725

726

727

728

729

730

731

732

733

734

735

736

737

738

739

[18] T. Wierzbicki, Y. Bao, Y. Lee, Y. Bai, Calibration and evaluation of seven fracture models, Int. J. Mech. Sci. 47 (2005) 719-743.

[19] ABAQUS, User's Manual, Version 6.12, Simulia, Providence, RI, 2012.

[20] G. Rousselier, Ductile fracture models and their potential in local approach of fracture, Nucl. Eng. Des. 105 (1987) 97-111.

[21] D.S. Liu, J.J. Lewandowski, The effects of superimposed hydrostatic pressure on deformation and fracture: Part II. Particulate-reinforced 6061 composites, Metall. Mater. Trans. A. 24 (1993) 609-615.

[22] H.A. Kuhn, P. Lee, Strain instability and fracture at the surface of upset cylinders, Metall. Mater. Trans. B. 2 (1971) 3197-3202.

[23] D. Vilotić, M. Plančak, Đ. Čupković, S. Alexandrov, N. Alexandrova, Free Surface Fracture in Three Upsetting Tests, Exp. Mech. 46 (2006) 115-120.

[24] N. Venkata Reddy, P.M. Dixit, G.K. Lal, Ductile fracture criteria and its prediction in axisymmetric drawing, Int. J. Mach. Tools Manuf. 40 (2000) 95-111.

[25] A. Gurson, Continuum theory of ductile rupture by void nucleation and growth: Part I-Yield criteria and flow rules for porous ductile media, J. Eng. Mater. (1977).

[26] Y. Huang, Accurate dilatation rates for spherical voids in triaxial stress fields, J. Appl. Mech. 58 (1991) 1990-1992.

[27] H. Kuwamura, K. Yamamoto, Ductile crack as trigger of brittle fracture in steel, J. Struct. Eng. 123 (1997) 729-735.

[28] Y. Bai, T. Wierzbicki, A new model of metal plasticity and fracture with pressure and Lode dependence, Int. J. Plast. 24 (2008) 1071-1096.

[29] C.M. Smith, A.M. Kanvinde, G.G. Deierlein, Stress-Weighted Damage Model for ductile fracture initiation in structural steel under cyclic loading and generalized stress states, Stanford, CA, 2013.

[30] A.M. Kanvinde, G.G. Deierlein, Micromechanical Simulation of EarthquakeInduced Fracture in Steel Structures, 2004.

[31] J. Hancock, D.K. Brown, On the role of strain and stress state in ductile failure, J. Mech. Phys. Solids. 31 (1983) 1-24.

[32] A. Argon, J. Im, R. Safoglu, Cavity formation from inclusions in ductile fracture, Metall. Trans. A. 6 (1975).

[33] A. Brownrigg, W. Spitzig, O. Richmond, D. Teirlinck, J.D. Embury, The influence of hydrostatic pressure on the flow stress and ductility of a spherodized 1045 steel, Acta Metall. 31 (1983) 1141-1150.

[34] M. Balzer, Mechanical behavior of metals under triaxial stress: apparatus and experiments, University of Illinois at Urbana-Champlain, 1998.

[35] A.A. Benzerga, J. Besson, a. Pineau, Anisotropic ductile fracture: Part I: Experiments, Acta Mater. 52 (2004) 4623-4638.

[36] T. Pardoen, F. Delannay, Assessment of void growth models from porosity measurements in cold-drawn copper bars, Metall. Mater. Trans. A. 29 (1998) 
741 [37] X. Gao, G. Zhang, C. Roe, A Study on the Effect of the Stress State on Ductile Fracture, Int. J. Damage Mech. 19 (2009) 75-94.

743 [38] C. Mcveigh, F. Vernerey, W. Liu, B. Moran, G.B. Olson, An interactive microvoid shear localization mechanism in high strength steels, J. Mech. Phys. Solids. 55 (2007) 225-244.

746 [39] J. Koplik, A. Needleman, Void growth and coalescence in porous plastic solids, Int. J. Solids Struct. (1988).

748 [40] W.-M. Chi, A.M. Kanvinde, G.G. Deierlein, Prediction of Ductile Fracture in Steel Connections Using SMCS Criterion, J. Struct. Eng. 132 (2006) 171.

750 [41] B. V Fell, A.M. Kanvinde, G.G. Deierlein, Experimental investigation of inelastic cyclic buckling and fracture of steel braces, J. Struct. Eng. (2009) 19-32.

752

753

754

755

756

[42] A.T. Myers, A.M. Kanvinde, G.G. Deierlein, J.W. Baker, Probabilistic Formulation of the Cyclic Void Growth Model to Predict Ultralow Cycle Fatigue in Structural Steel, J. Eng. Mech. 140 (2014) 04014028.

757

[43] J.R. Benjamin, C.A. Cornell, Probability, Statistics, and Decisions for Civil Engineering, McGraw-Hill Book Co., New York, 1970.

758 


\begin{tabular}{|c|c|c|c|c|c|}
\hline \multicolumn{2}{|r|}{ Model } & \multicolumn{4}{|c|}{ Log-likelihood by supporting data set } \\
\hline Source & Fracture criteria & $\begin{array}{c}\text { Analytical } \\
\text { void } \\
\text { growth } \\
{[26]} \\
\end{array}$ & $\begin{array}{c}\text { FEM -void } \\
\text { coalescence } \\
{[15]}\end{array}$ & $\begin{array}{l}\text { CNT } \\
\text { tests } \\
{[11]}\end{array}$ & $\begin{array}{c}\text { High- } \\
\text { pressure } \\
\text { tension } \\
\text { tests [5] }\end{array}$ \\
\hline $\begin{array}{l}\text { Proposed (Eq. } \\
\text { 18) }\end{array}$ & $D=\int e^{1.3 T}-e^{-1.3 T} d \varepsilon_{P}$ & 0.0 & 0.0 & 0.0 & 0.0 \\
\hline VGM [2] & $D=\int e^{1.5 T} d \varepsilon_{P}$ & 1.2 & -2.7 & -6.8 & -12.9 \\
\hline SMCS [4] & $\varepsilon_{p, \text { crit }}=\alpha e^{-1.5 T}$ & 1.2 & -2.7 & -23.6 & -135.2 \\
\hline $\begin{array}{l}\text { Kuwamura and } \\
\text { Yamamoto [27] }\end{array}$ & $\varepsilon_{p, c r i t}=C T^{-2}$ & -405.6 & -9.1 & 1.0 & -83.4 \\
\hline $\begin{array}{l}\text { Bao and } \\
\text { Wierzbicki [4] }\end{array}$ & $\begin{array}{l}\varepsilon_{p, \text { crit }}= \\
\left\{\begin{array}{cc}T<0 & C\left(T+\frac{1}{3}\right)^{-1} \\
0<T<\frac{1}{3} & \ldots \\
T>\frac{1}{3} & C T^{-1}\end{array}\right.\end{array}$ & -576.8 & -16.3 & -4.0 & -62.2 \\
\hline
\end{tabular}

Figure Captions:

764 Figure 1: Proposed relationship between fracture damage rate and stress triaxiality

Figure 1: Ultimate conditions in smooth notched bars tested by Bridgman [1] Footnote:

${ }^{1}$ Fracture observations at strains above $\overline{\varepsilon_{p}}=3.5$ are deemed unreliable due to near-complete area reduction of specimens by the time of failure

Figure 3: Schematic history of continuum parameters in Bridgman specimens

Figure 4: Conditions at fracture in tension coupons and CNT specimens

Figure 5: Damage rate of the proposed criteria compared with void growth predictions of Rice and Tracey [6] and Huang [26]

Figure 6: Damage rate of the proposed criteria fit to void growth measurements from single void cell simulations by Cooke et al. [15] six materials under varying stress triaxiality, (b) bulk stress-strain response of voided material illustrating the determination of the localization strain. Adapted from Cooke [15]

779 Figure 8: Plastic strain and calculated damage history for a hypothetical steel

780 Figure 9: Likelihood of models with varying fracture cutoffs

781 Figure 10: Likelihood of models with varying $A$ parameters 


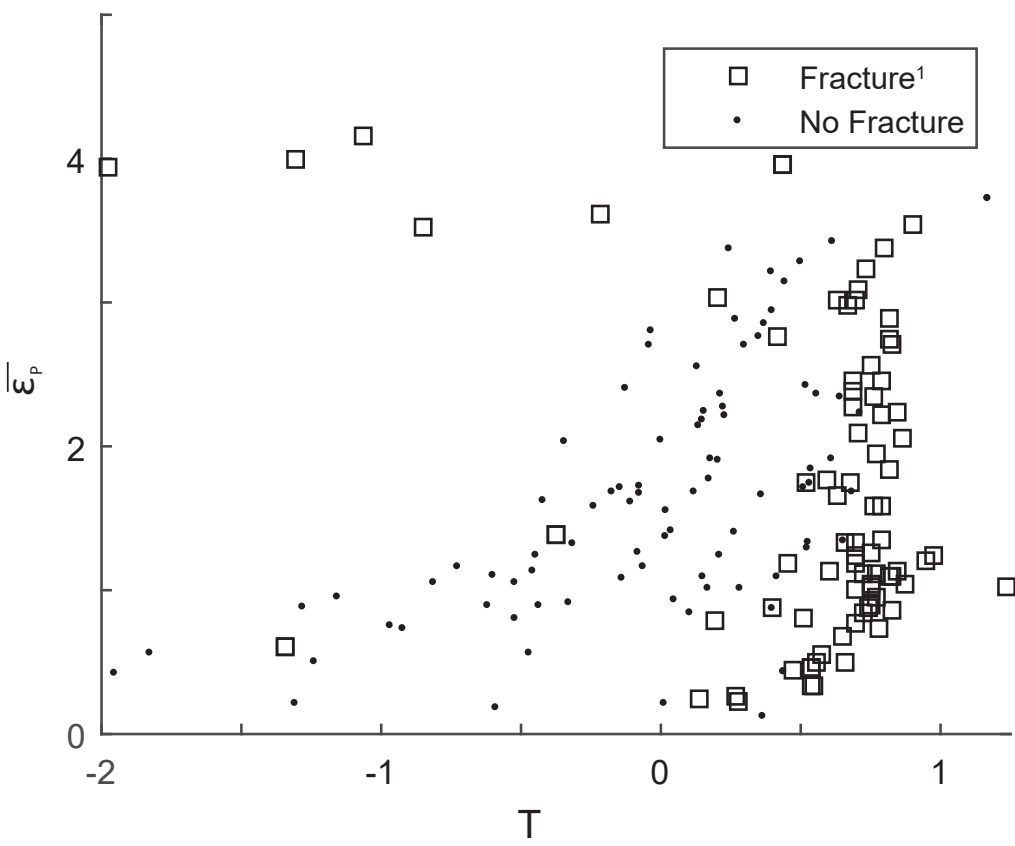




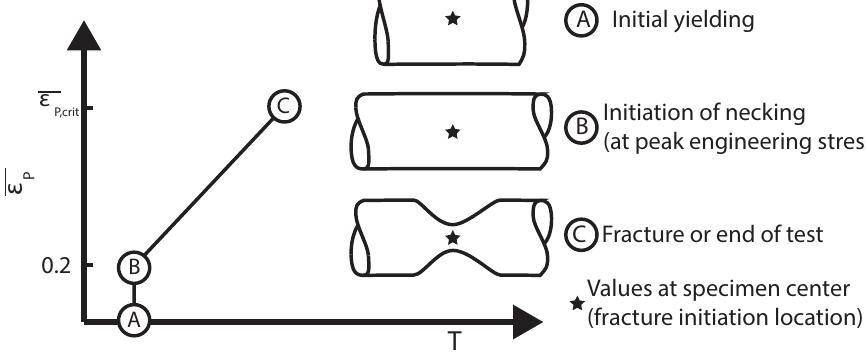




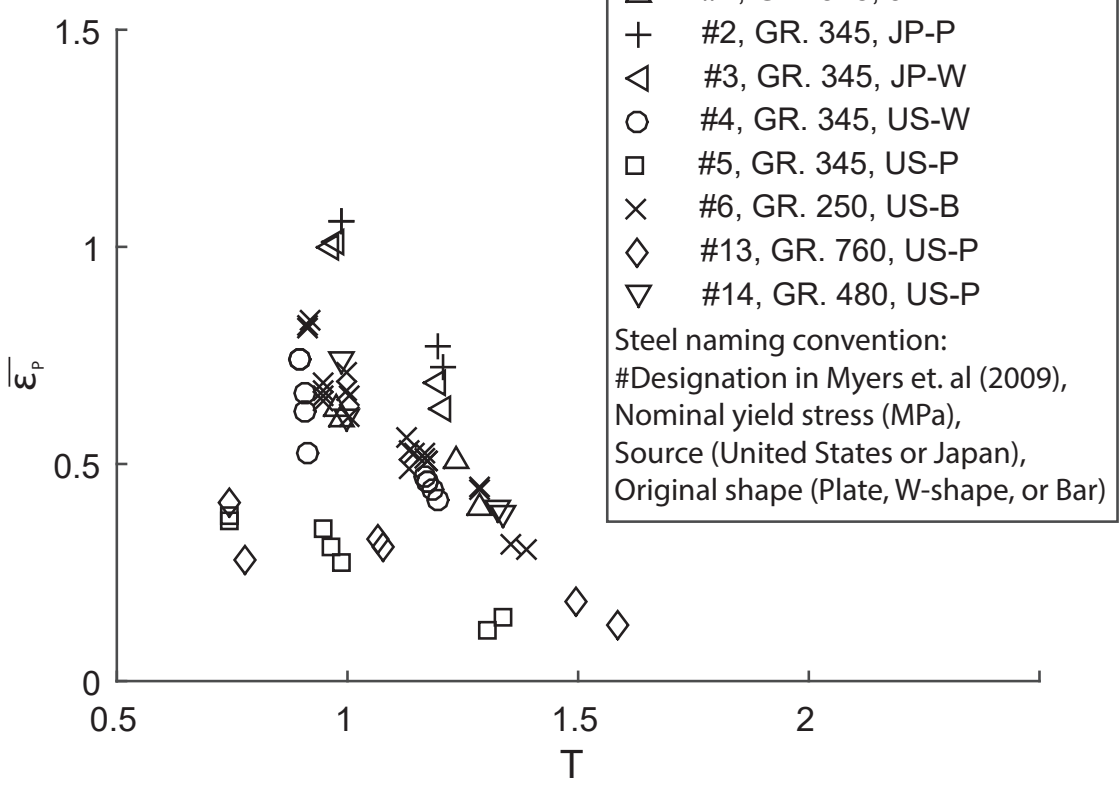




\section{\begin{tabular}{|l|l} 
Equation 1 (Rice and Tracey 1969) \\
\hline
\end{tabular} \\ - Equation 5 (Huang 1991) \\ Equation 9 (Proposed damage rate)}

$\oslash \mid \frac{\omega}{0}$

0

1

2 


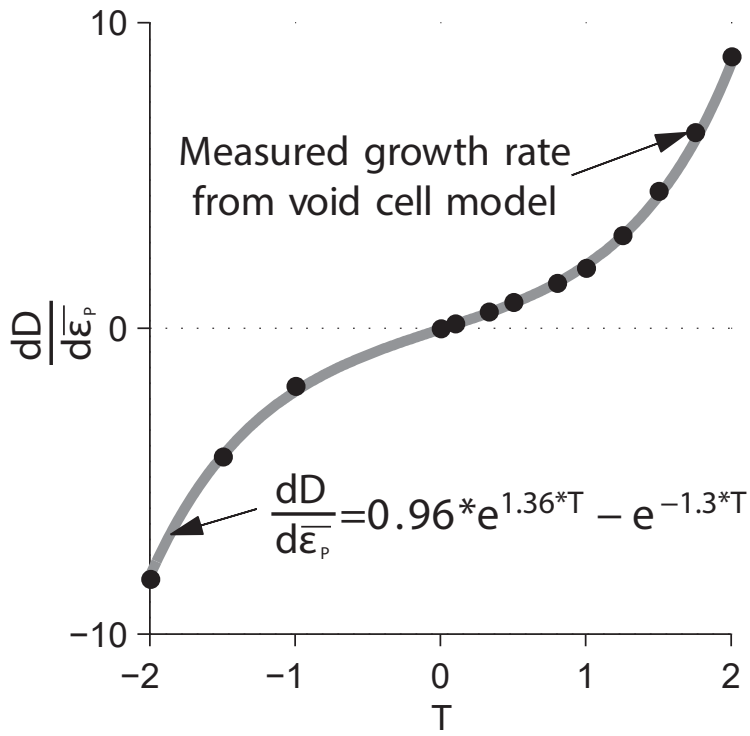



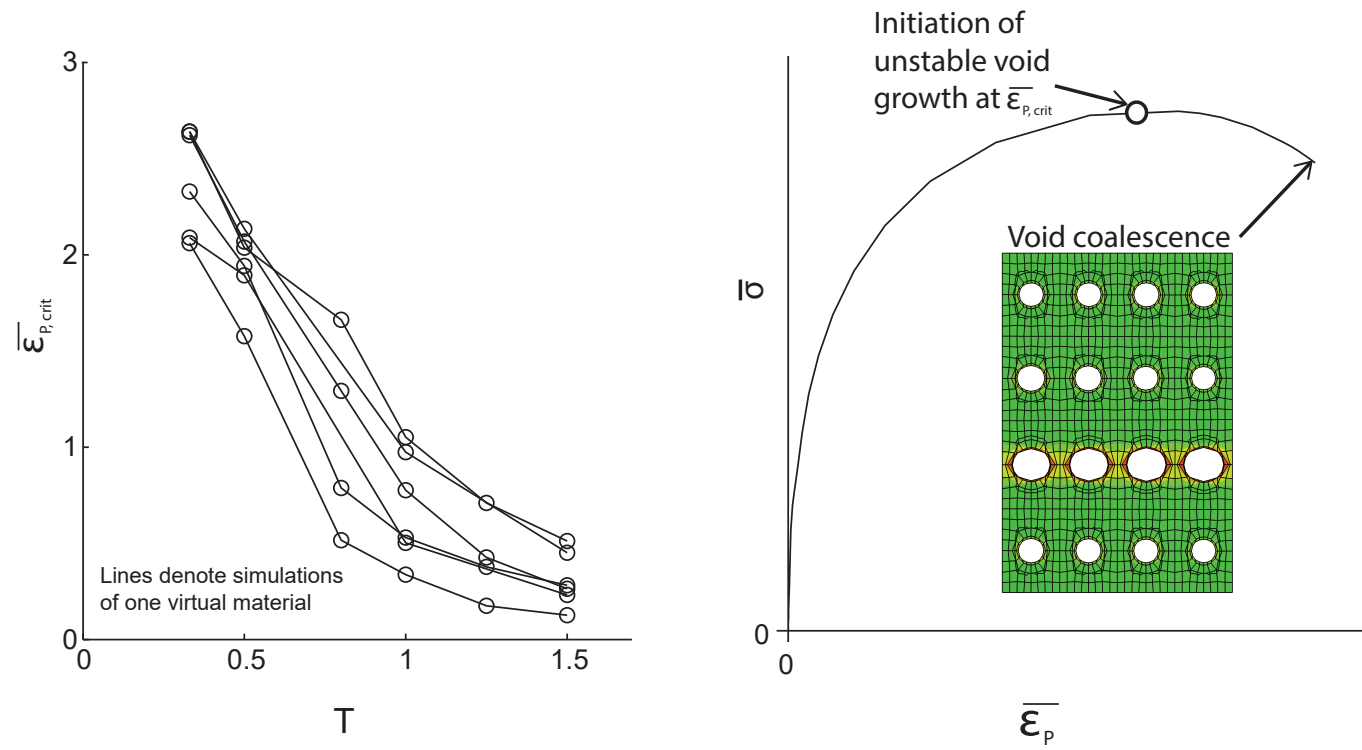

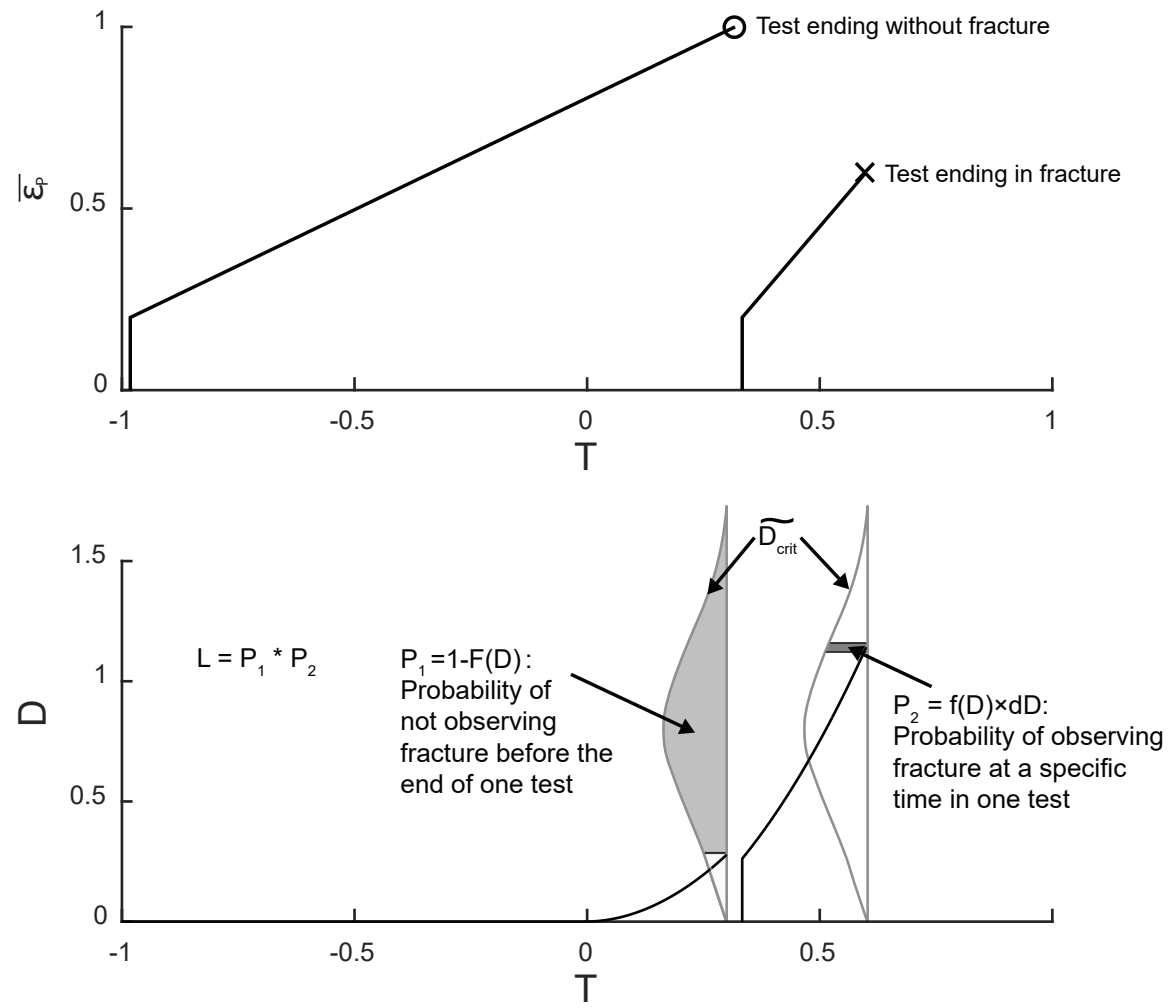
Cutoff Triaxiality

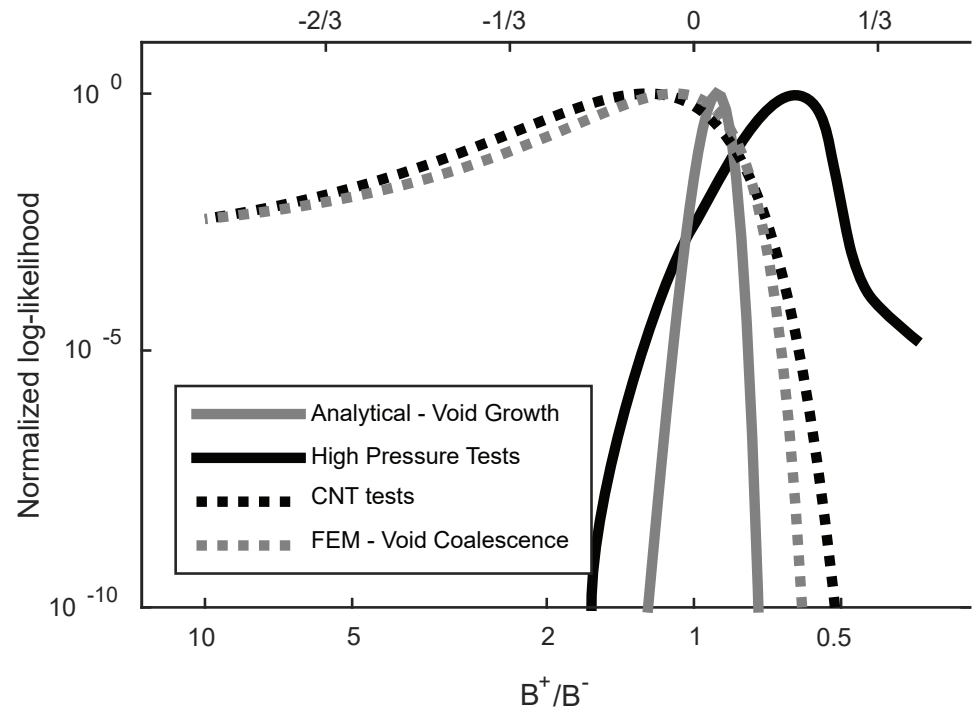


